\title{
Systemic inflammatory markers and serum lactate dehydrogenase predict survival in patients with Wilms tumour
}

\author{
Michał Kunc ${ }^{1}$, Anna Gabrych², Dominika Dulak³, Karolina Hasko³, Malgorzata Styczewska ${ }^{3}$, \\ Dagmara Szmyd ${ }^{4}$, Kristoffer Nilsson ${ }^{3}$, Marek Iwinski ${ }^{3}$, Agata Sobocińska-Mirska ${ }^{5}$, \\ Malgorzata Sawicka-Zukowska ${ }^{6}$, Malgorzata A. Krawczyk², Ewa Bien²
}

\author{
${ }^{1}$ Department of Pathomorphology, Medical University of Gdansk, Gdansk, Poland \\ 2Department of Pediatrics, Hematology and Oncology, Medical University of Gdansk, \\ Gdansk, Poland \\ ${ }^{3}$ The English Division Pediatric Oncology Scientific Circle, Medical University of \\ Gdansk, Gdansk, Poland \\ ${ }^{4}$ Coronary Care Unit, Cardiology Department, West Cumberland Hospital, Whitehaven, \\ United Kingdom \\ ${ }^{5}$ Department of Pediatrics, Oncology and Hematology, Medical University of Warsaw, \\ Warsaw, Poland \\ ${ }^{6}$ Department of Pediatrics, Paediatric Oncology, and Hematology, Medical University \\ of Bialystok, Bialystok, Poland
}

Submitted: 16 April 2020; Accepted: 19 July 2020

Online publication: 18 April 2021

Arch Med Sci 2022; 18 (5): 1253-1261

DOI: https://doi.org/10.5114/aoms/125543

Copyright (c) 2021 Termedia \& Banach

\begin{abstract}
Introduction: Markers of inflammation such as neutrophil-to-lymphocyte ratio (NLR), platelet-to-lymphocyte ratio (PLR), and lymphocyte-to-monocyte ratio (LMR) have been found to be associated with survival in cancer patients. The aim of the current study was to establish the prognostic significance of simple laboratory markers of systemic inflammation in paediatric patients diagnosed with Wilms tumour (WT). Additionally, we aimed to compare the complete blood count (CBC) parameters of WT patients and the non-oncological control group.

Material and methods: The study group included 88 children diagnosed with WT. Clinicopathological data, as well as CBC, C-reactive protein (CRP) and lactate dehydrogenase (LDH) levels at diagnosis, were obtained. Additionally, the laboratory results of 62 healthy control paediatric patients were collected. Uni- and multivariate proportional Cox's hazard analyses were computed to create a model predicting relapse-free survival (RFS) and overall survival (OS) in the study group.

Results: High CRP, LDH, and NLR were associated with a higher stage of WT and shorter RFS, whereas all parameters correlated with OS. In multivariate analysis, only LDH levels had adverse significance in predicting RFS. C-reactive protein and $L M R$ retained their prognostic value in the multivariate model predicting OS. Comparing the WT group with controls, high LDH, high CRP, high NLR, and high PLR were associated with WT presence.

Conclusions: Preoperative LDH, CRP, NLR, PLR, and LMR have significant prognostic value in patients with WT independently of age and stage. Combined low CRP and high LMR identified the group of patients with excellent OS. Patients with high LDH were characterized by the highest risk of relapse.
\end{abstract}

Key words: neutrophils, systemic inflammation, lactate dehydrogenase, paediatric, Wilms tumour.
Corresponding author:

Prof. Ewa Bien

Department of Pediatrics, Hematology and Oncology Medical University

of Gdansk

Gdansk, Poland

E-mail: ewa.bien@gumed. edu.pl 


\section{Introduction}

Nephroblastoma, also called Wilms tumour (WT), is the most common paediatric renal tumour. The highest incidence of WT occurs in children younger than 5 years of age. Occasionally, older children or adults are also affected. The management of WT has changed substantially over the last 50 years, resulting in high cure rates, reaching $90 \%$ in localized tumours [1]. However, the treatment outcomes in children with WT stage III and IV and/or unfavourable tumour histology remain unsatisfactory [2].

Current therapeutic options in WT include chemotherapy $(\mathrm{CHT})$, surgery and radiotherapy. Such a multimodal treatment of WT, although highly efficacious, is associated with significant late effects, including chronic renal insufficiency, secondary tumours, cardiac toxicity, impaired growth, infertility, and end-stage renal failure, particularly in bilateral WT $[3,4]$.

To reduce the risk of late complications, the intensity of therapy should be precisely adjusted to the risk factors present in particular patients [5]. However, the treatment strategy in WT according to the International Society of Paediatric Oncology (SIOP) assumes starting neoadjuvant $\mathrm{CHT}$, based on characteristic clinical and radiological features, without performing tumour biopsy. Histology of WT (favourable or unfavourable) and the disease stage become known after the tumour resection during nephrectomy. Therefore, the risk stratification in children with WT is possible only after pre-operative $\mathrm{CHT}$ and surgery.

It would be of great clinical benefit to be able to assess the risk of relapse and poor outcome earlier, preferably at diagnosis of WT. However, no reliable biochemical markers of WT have been determined to date. Thus, searching for new, easily measured biochemical prognostic markers that might play a role in the management of patients with nephroblastoma is necessary.

In recent years, there has been increasing interest in complete blood count (CBC) parameters used as prognostic factors in various malignancies. The data suggest that inflammation plays a crucial role in tumour formation [6]. A tumour-associated inflammatory response may promote invasion, angiogenesis and distant metastases [7] Elevated inflammatory markers have been found to play roles of adverse prognostic factors in various malignancies, including colorectal, rectal, gastric, prostate and breast cancer [8-13]. Complete blood count is an inexpensive and readily available test in daily practice that can indirectly reflect the degree of inflammation. The values of neutrophil-to-lymphocyte ratio (NLR), platelet-to-lymphocyte ratio (PLR), and lymphocyte-to-monocyte ratio (LMR) have emerged as promising tools to predict outcomes in common malignancies of adulthood, including breast, lung and colorectal cancers [14-16]. Recently it has been proven that a similar association exists in a paediatric population. Prognostic value of NLR has been demonstrated in children with neuroblastoma and other paediatric solid tumours [17], malignancies of salivary glands [18], soft tissue sarcomas [19], and brain tumours [20].

To our knowledge, no study to date has analysed the particular association between $C B C$ ratios and prognosis in patients with WT.

The current study aims to establish the correlation between simple laboratory parameters of inflammation, derived from CBC, and the relapse-free survival (RFS) and overall survival (OS) in a cohort of patients with WT treated in three Polish centres of paediatric oncology within the last 20 years. Our aim is also to determine whether CBC-derived markers might help to stratify the patients with WT to the risk groups before systemic treatment starts. The secondary aim is to find out if the $C B C$ parameters may distinguish patients with WT from non-oncological patients.

\section{Material and methods}

The study group consisted of patients diagnosed with WT between 2002 and 2019 at the Department of Paediatrics, Haematology, and Oncology in Gdansk, the Department of Paediatric Oncology and Haematology in Bialystok, and the Department of Paediatrics, Oncology, and Haematology, Medical University of Warsaw, Poland. In all cases, the histopathologic diagnoses of WT were verified at two independent reference centres, and the patients were treated according to the same treatment protocol. Age at presentation, gender, $\mathrm{CBC}$ at the time of diagnosis, value of $\mathrm{C}$-reactive protein (CRP), serum lactate dehydrogenase (LDH) level, WT stage, date of relapse and death were retrieved from each patient's medical record. Patients with bone marrow involvement, concomitant haematological diseases, active infection, haemolysis of blood samples and incomplete laboratory or follow-up data $(n=71)$ were excluded from the study.

Additionally, the $C B C$ results of 63 control paediatric patients without malignancy were collected. These patients had been initially suspected of malignancy, which was ruled out in the further diagnostics performed at the Department of Paediatrics, Haematology, and Oncology in Gdansk. The control group included children diagnosed with: testicular microlithiasis $(n=24)$, benign bone or joint lesions $(n=9)$, benign adrenal lesions $(n=5)$, non-malignant cutaneous or soft tissue lesions $(n=4)$, CNS abnormalities $(n=3)$, neurofibromatosis type $1(n=2)$, ovarian abnormalities $(n=2)$, and 
other $(n=14)$. Patients with any haematological diseases, ongoing infection or any inflammatory conditions were excluded from the control group.

Taking into consideration the physiological changes in $C B C$ in children during ageing, the norms for each analysed parameter were assessed according to the patient age using the National Health Service Children's Reference Ranges for Routine Haematology Tests. Then, the absolute neutrophil count was divided by the upper cut-off value of the norm (appropriate for the age) resulting in obtaining a ratio of neutrophils compared to the upper norm. The same procedure was performed for monocytes and platelets. The lymphocyte counts were compared to a lower cut-off value of the norm because lymphopenia is a risk factor for shorter RFS and OS in cancer patients [21]. Subsequently, the obtained parameters were used to calculate LMR, NLR, and PLR comparedto-norm values. If not otherwise specified, in the following text, LMR, NLR, PLR parameters are age-adjusted.

\section{Statistical analysis}

Statistical analysis was performed with Statistica 13 (Dell, USA) licensed to the Medical University of Gdansk. Receiver operating curves (ROC) were created for NLR, LMR, PLR, LDH, and CRP. Youden's index was employed to estimate the optimal cut-off values for the relationship with relapse and death separately. Another ROC was constructed to determine the cut-off values discriminating patients with WT and controls. Uniand multivariate proportional Cox's hazard analyses were computed to create a model predicting RFS and OS in the study group. The variables with $p<0.1$ in univariate analysis were included in multivariate analysis. The Kaplan-Meier curves with the log-rank test were plotted to estimate the OS and RFS. Overall survival was defined as the time between the diagnosis and death, and RFS was defined as the period between the diagnosis and the first recurrence. The correlations between continuous variables were tested with Spearman's correlation, whereas the associations between categorical variables were analysed with $\chi^{2}$ or Fisher's test when applicable. Univariate logistic regression was utilized to examine the predictive value of laboratory counts for the presence of WT. $P$-values $<0.05$ were considered statistically significant.

\section{Results}

Eighty-eight patients were finally enrolled in the study, including 43 females and 45 males. At the time of diagnosis, the mean age was 4.00 years, with a median of 3.45 years, and age range between 0 and 15.51 years. The mean follow-up was 5.75 years with a median of 4.96 years and range between 0.33 and 15.4 years. There were $41,18,15,8,6$ patients with stage I, II, III, IV and V of WT, respectively. In the entire study, there were 5 disease-related deaths and 12 cases of relapse of WT.

The values of $L M R$ and PLR compared to the norm correlated significantly with age (Spearman's rho coefficient: -0.31 and 0.11 , respectively), but this effect was smaller than for non-normalized LMR and PLR (Spearman's rho coefficient: -0.47 and 0.48, respectively). Age-adjusted NLR was not associated with age (Spearman's rho coefficient: $0.12, p=0.244)$, in contrast to non-adjusted NLR (Spearman's rho coefficient: 0.38, $p=0.0002$ ) (Figures $1 \mathrm{~A}, \mathrm{~B})$. Additionally, high CRP, LDH, and NLR were associated with higher stage $(p=0.001, p=$ $0.033, p=0.018$, respectively; $\chi^{2}$ test).

ROCs for every variable (LDH, CRP, NLR, LMR, and PLR) are shown in Figures 2 A, B, separately for relapse and death respectively, and area under curve (AUC) values are shown in Table I. Area under curve for LDH was significantly higher than for PLR in case of relapse $(p=0.015)$. The 5-year RFS and 5-year OS calculated for each parameter (high- vs. low) based on Kaplan-Meier curves (Figures 3 and 4) are presented in Table II. High LDH, CRP, and NLR were significantly associated with RFS, whereas all parameters correlated with OS.

In the next step, Cox logistic regression analysis was employed to create a multivariate model to predict survival in WT patients. Only LDH serum levels had independent adverse significance after multivariate analysis in predicting RFS (Table III), and the patients with high LDH exhibited the highest relapse rate. On the other hand, CRP and $L M R$ retained their prognostic value in the model predicting OS (Table IV). In the group of patients displaying both low CRP and high LMR there was $100 \%$ 5-year OS ( $p=0.00134$, log-rank).

The control group consisted of 42 males and 20 females. The mean age at admission was 7.29 years (median age: 6.35 years; range: 0-17.7 years). The screening for malignancy was negative in all patients. Thus, our next aim was to establish whether systemic inflammatory markers may discriminate WT patients from non-oncological patients from the control group.

For this purpose, other ROCs were plotted (Figure $1 \mathrm{C}$ ), and AUCs were calculated (Table I). The AUC of LDH was significantly higher than that of all other variables $(p<0.05)$. The results of univariate logistic regression analysis showed that high $\mathrm{LDH}(\mathrm{OR}=36.34,95 \% \mathrm{Cl}: 13.85-95.37 ; p<0.001)$, high CRP $(\mathrm{OR}=8.575,95 \% \mathrm{Cl}: 3.83-19.22, p<$ $0.001)$, high NLR (OR $=8.29,95 \% \mathrm{Cl}: 2.75-24.95)$, and high PLR $(\mathrm{OR}=2.96,95 \% \mathrm{Cl}: 1.50-5.82)$ were 
M. Kunc, A. Gabrych, D. Dulak, K. Hasko, M. Styczewska, D. Szmyd, K. Nilsson, M. Iwinski, A. Sobocińska-Mirska, M. Sawicka-Zukowska, M.A. Krawczyk, E. Bien

A

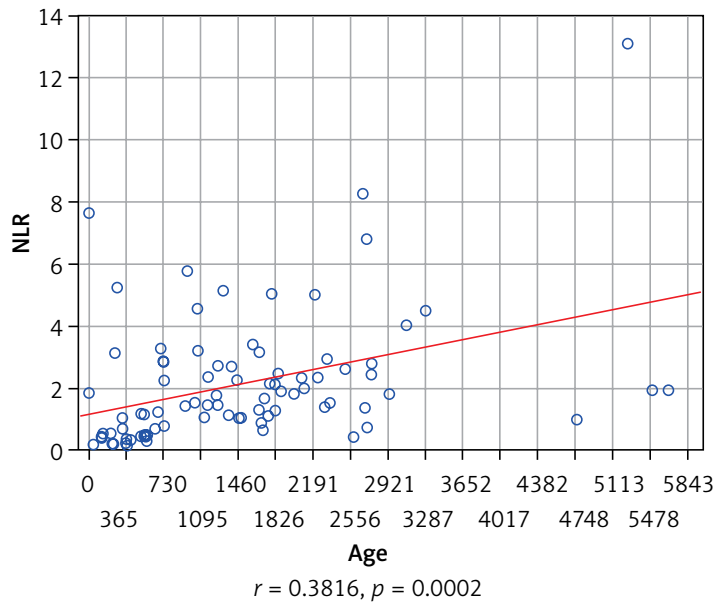

B

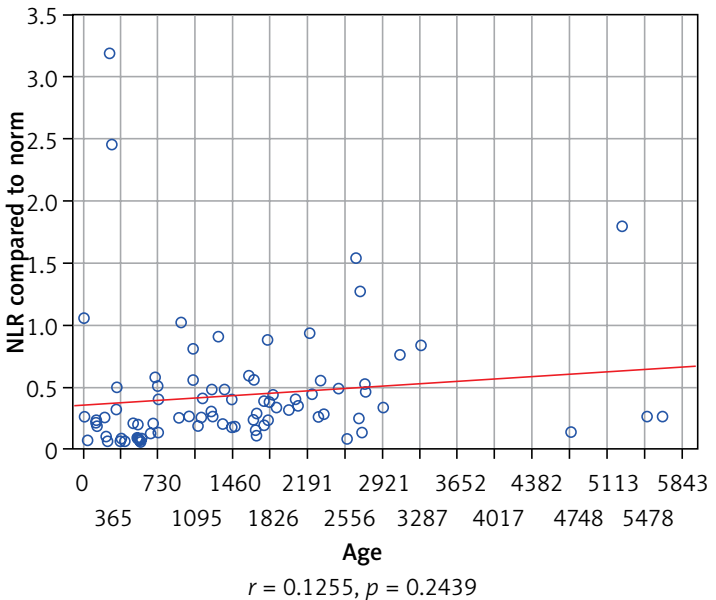

Figure 1. Correlation of neutrophil-to-lymphocyte ratio (NLR) (A) and NLR compared to norm (B) with age in days. The Spearman correlation coefficient was calculated to measure the strength of association

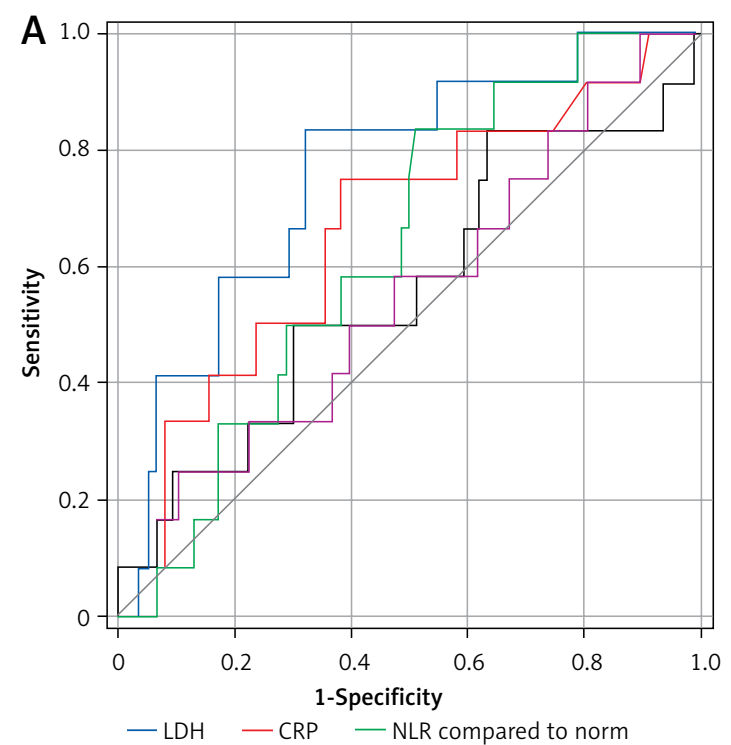

- PLR compared to norm _ LMR compared to norm - Reference line

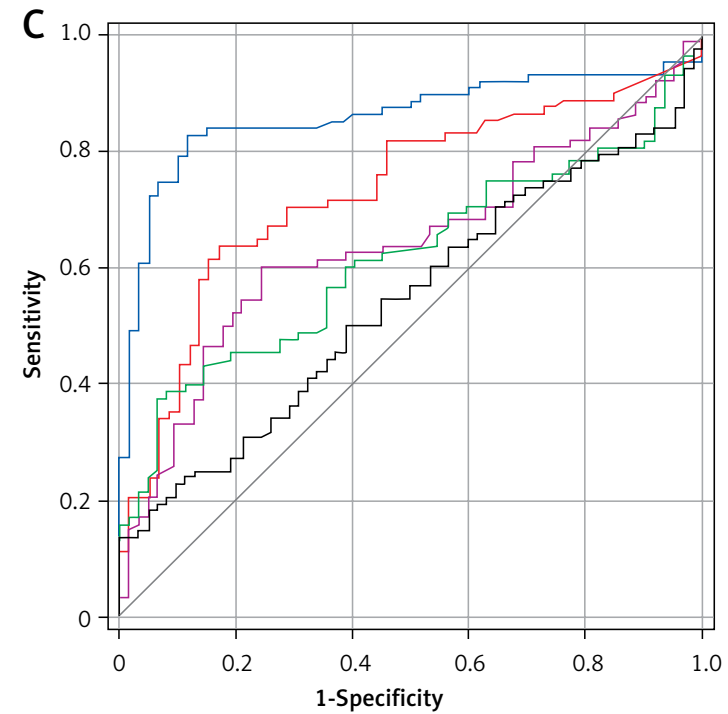

- LDH - CRP — NLR compared to norm

- PLR compared to norm — LMR compared to norm - Reference line

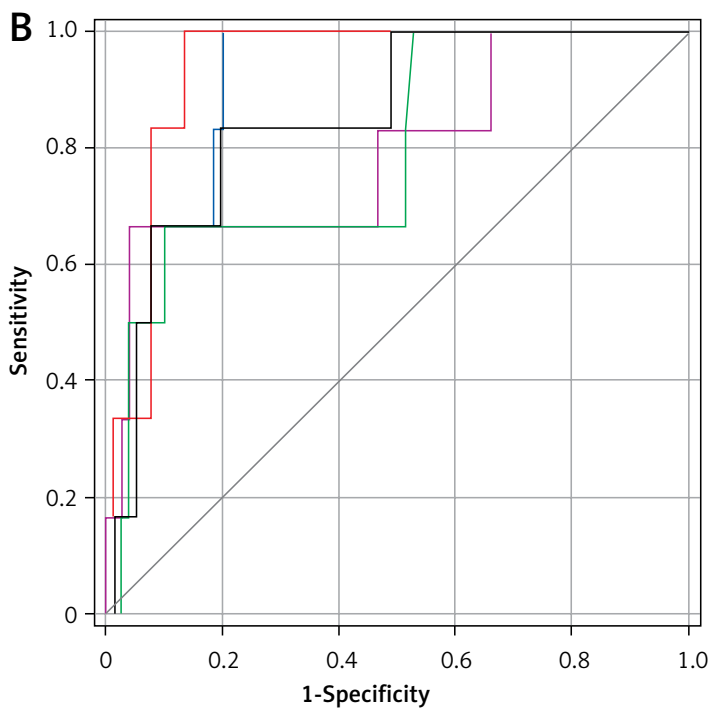

- $\mathrm{LDH}$ - CRP — NLR compared to norm

- PLR compared to norm - LMR compared to norm - Reference line

Figure 2. Receiver operating curves for each parameter plotted for relapse (A), death (B) and differentiation between Wilms tumour (WT) and controls (C) 
Table I. Areas under curves for investigated markers

\begin{tabular}{|lcccccc|}
\hline Variable & $\begin{array}{c}\text { AUC } \\
\text { (death) }\end{array}$ & $\begin{array}{c}\text { Cut-off } \\
\text { (death) }\end{array}$ & $\begin{array}{c}\text { AUC } \\
\text { (relapse) }\end{array}$ & $\begin{array}{c}\text { Cut-off } \\
\text { (relapse) }\end{array}$ & $\begin{array}{c}\text { AUC } \\
\text { (vs. controls) }\end{array}$ & $\begin{array}{c}\text { Cut-off } \\
\text { (vs. controls) }\end{array}$ \\
\hline LDH [U/l] & 0.90 & 896 & 0.75 & 731 & 0.83 & 322 \\
\hline CRP $[\mathrm{mg} / \mathrm{dl}]$ & 0.93 & 25.02 & 0.67 & 5.5 & 0.70 & 1.950 \\
\hline NLR & 0.80 & 0.808 & 0.63 & 0.262 & 0.35 & 0.387 \\
\hline PLR & 0.80 & 1.211 & 0.55 & 0.928 & 0.36 & 0.490 \\
\hline LMR & 0.86 & 1.611 & 0.56 & 3.293 & 0.43 & 1.170 \\
\hline
\end{tabular}

$L D H$ - lactate dehydrogenase, CRP - C-reactive protein, NLR - neutrophil-to-lymphocyte ratio, PLR - platelet-to-lymphocyte ratio, $L M R$ - lymphocyte-to-monocyte ratio.

A

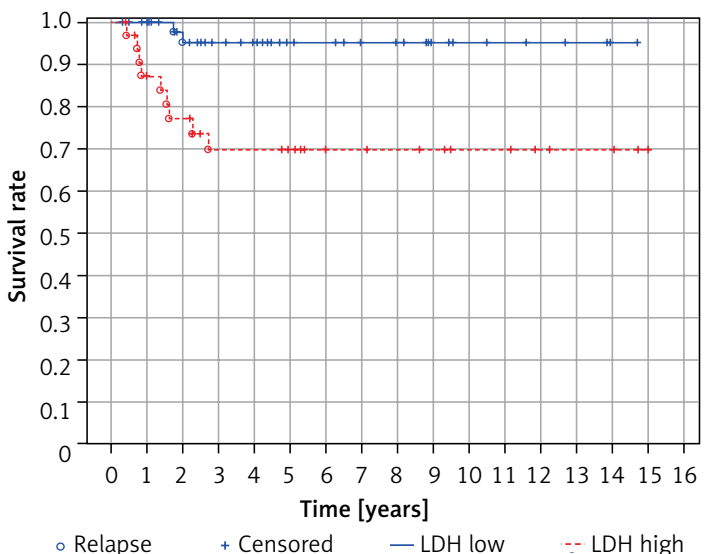

C

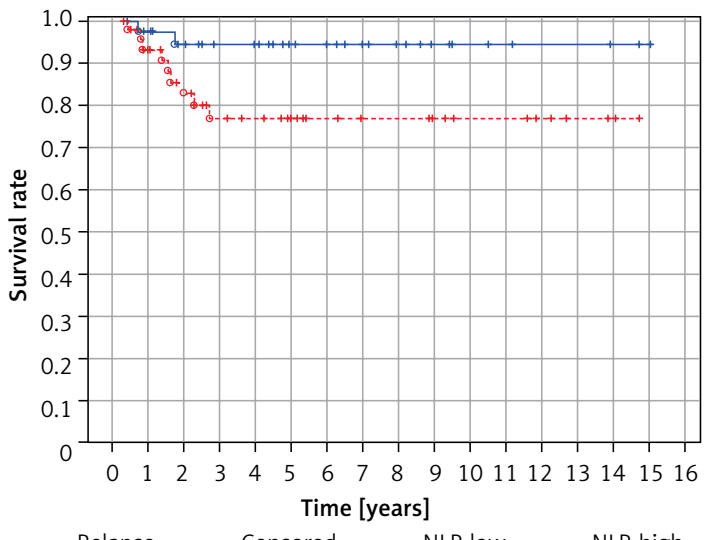

B

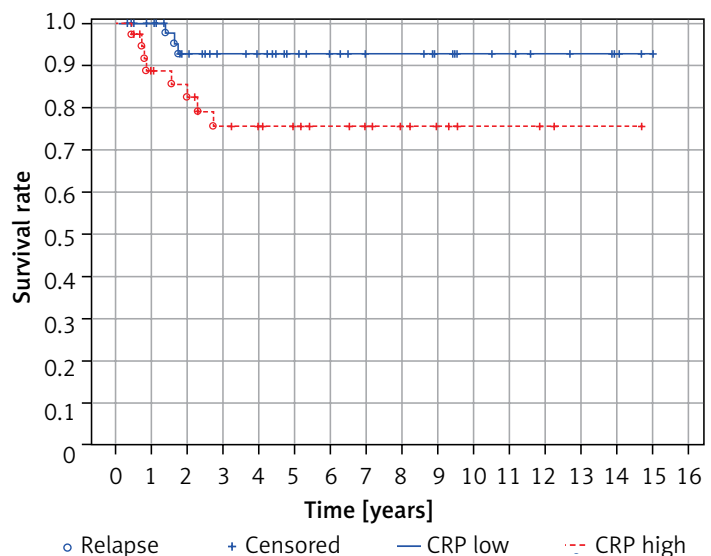

D

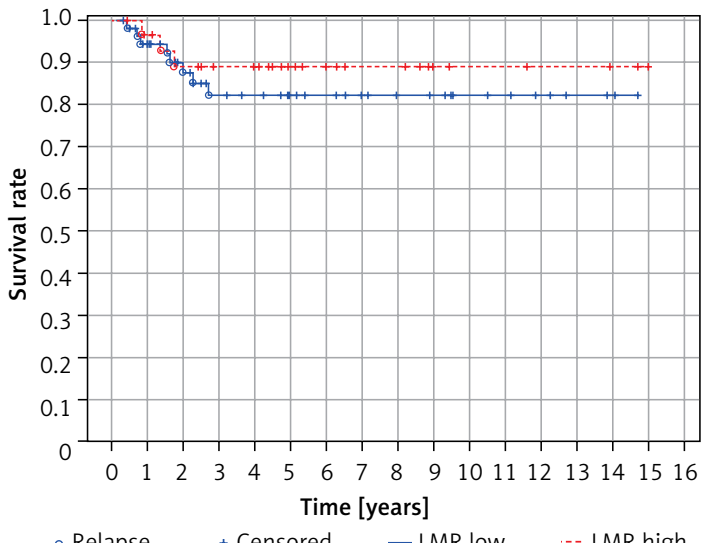

Figure 3. Kaplan-Meier curves for relapse-free survival plotted for patients with low or high level of the selected biomarkers

associated with WT presence. Only LMR did not demonstrate any value to differentiate between WT patients and controls.

\section{Discussion}

Measuring CBC parameters, such as neutrophils, lymphocytes and platelets and determining the respective ratios of selected CBS parameters might help in prognostication of some neoplastic and non-neoplastic diseases. One recent study demonstrated that NLR aids predicting complications in children with acute appendicitis [22]. In most studies published to date, cancer patients exhibiting high NLR, high PLR and low LMR showed worse outcomes. Elevated levels of neutrophils, platelets, and monocytes compared to lymphocytes might be a consequence of the systemic inflammatory reaction in response to the presence of malignancy. The latter may support cancer progression, finally contributing to the worse survival of cancer patients.

Neutrophils are well known cells involved in systemic inflammation. They produce cytokines and angiogenic factors that promote tumour progression, whereas lymphocytes contribute mainly 
M. Kunc, A. Gabrych, D. Dulak, K. Hasko, M. Styczewska, D. Szmyd, K. Nilsson, M. Iwinski, A. Sobocińska-Mirska, M. Sawicka-Zukowska, M.A. Krawczyk, E. Bien

A

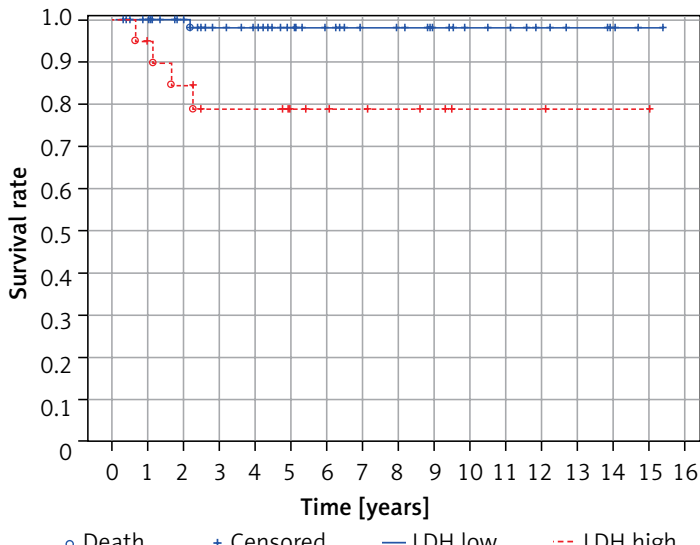

C

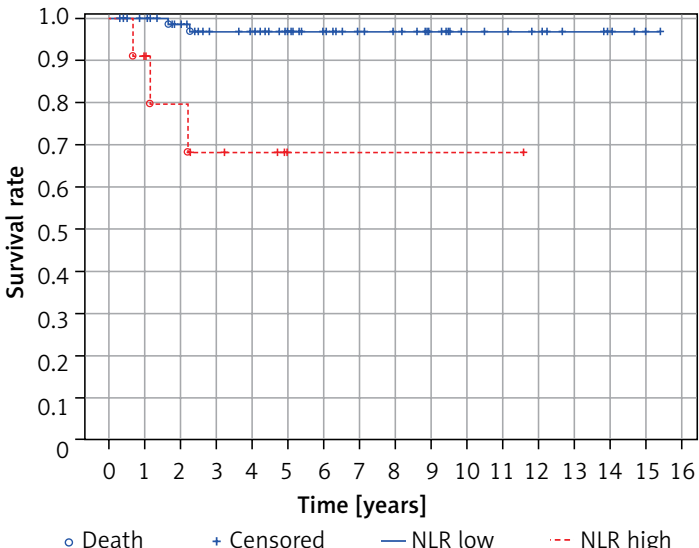

E

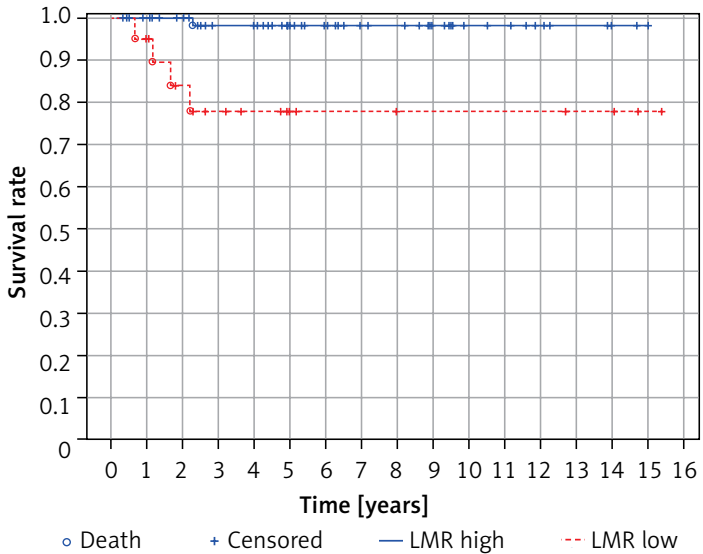

B

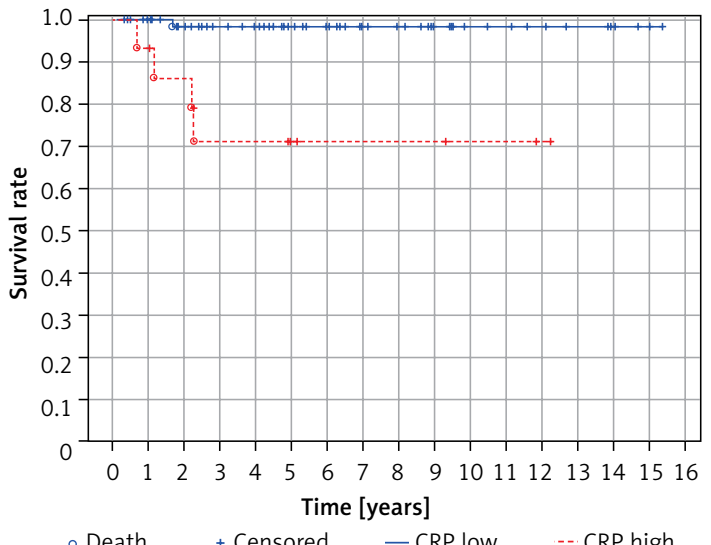

D

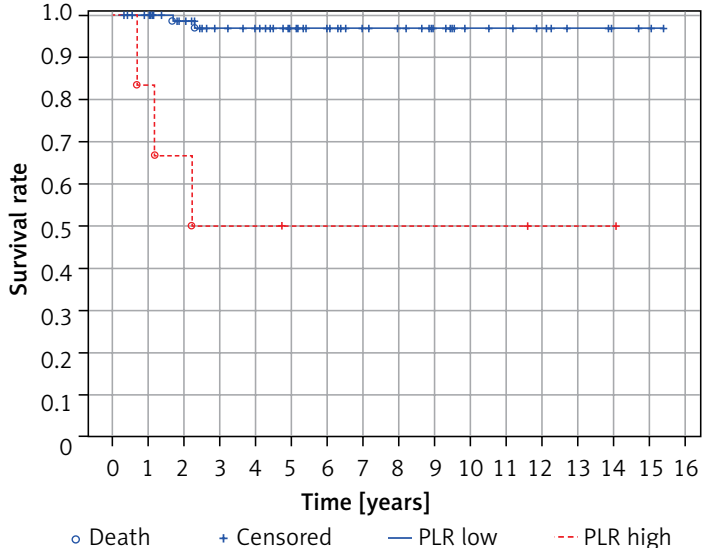

$\mathrm{F}$

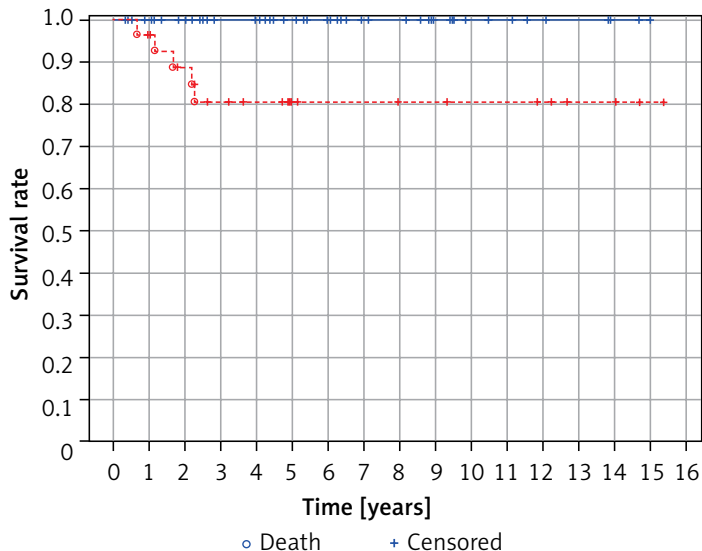

—CRP low and LMR high $\quad$-. CRP high and/or LMR low

Figure 4. Kaplan-Meier curves for overall survival plotted for patients with low or high level of the selected biomarkers

to the process of elimination of cancer cells [23]. Thus, a disproportion in these blood components might have an impact on tumour growth and progression [24].

The data regarding the role of the systemic inflammatory response in WT patients remain very sparse. Recently, the local tumour microenvironment has gained more attention, and various pop- ulations of immune cells, including tumour-associated macrophages, tumour-associated neutrophils, and tumour-associated lymphocytes, have been described in WTs [25]. Interestingly, up-regulation of the cyclooxygenase 2 pathway enriches the tumour with immunosuppressive inflammatory cells, suggesting that tumour-promoting inflammation may play a role in progression of WT [26]. 
Table II. Comparison of survival between groups with low and high expression of investigated markers. $P$-values calculated with log-rank test

\begin{tabular}{|c|c|c|c|c|c|c|}
\hline \multirow[t]{2}{*}{ Variable } & \multicolumn{2}{|c|}{5 year RFS (\%) } & \multirow[t]{2}{*}{$P$-value } & \multicolumn{2}{|c|}{5 year OS (\%) } & \multirow[t]{2}{*}{$P$-value } \\
\hline & Low & High & & Low & High & \\
\hline LDH & 95.23 & 69.75 & 0.002 & 98.04 & 78.81 & 0.003 \\
\hline CRP & 93.00 & 75.36 & 0.037 & 98.33 & 71.08 & $<0.001$ \\
\hline NLR & 94.42 & 76.69 & 0.043 & 68.31 & 96.79 & $<0.001$ \\
\hline PLR & 84.72 & 87.50 & 0.929 & 96.91 & 50.00 & $<0.001$ \\
\hline LMR & 82.22 & 89.08 & 0.461 & 77.84 & 98.09 & 0.002 \\
\hline
\end{tabular}

$L D H$ - lactate dehydrogenase, CRP - C-reactive protein, NLR - neutrophil-to-lymphocyte ratio, PLR - platelet-to-lymphocyte ratio, $L M R$ - lymphocyte-to-monocyte ratio.

Table III. Uni- and multivariate Cox regression analysis for relapse-free survival in patients with Wilms tumour

\begin{tabular}{|lcccc|}
\hline Characteristics & $\begin{array}{c}\text { Univariate } \\
\text { HR }(95 \% \mathrm{Cl})\end{array}$ & P-value & $\begin{array}{c}\text { Multivariate } \\
\text { HR }(95 \% \mathrm{Cl})\end{array}$ & $P$-value \\
\hline Age $(<4$ y vs. > 4 y) & $0.92(0.28-3.03)$ & 0.897 & & \\
\hline Stage (4-5 vs. $1-3)$ & $1.26(0.37-4.32)$ & 0.708 & & \\
\hline LDH (H vs. L) & $7.75(1.67-35.87)$ & 0.009 & $7.75(1.67-35.87)$ & \\
\hline CRP (H vs. L) & $3.73(0.99-14.08)$ & 0.052 & \\
\hline NLR (H vs. L) & $4.25(0.91-19.71)$ & 0.064 & \\
\hline PLR (H vs. L) & $1.57(0.20-12.30)$ & 0.666 & & \\
\hline LMR (L vs. H) & $1.63(0.43-6.14)$ & 0.472 & & \\
\hline
\end{tabular}

$L D H$ - lactate dehydrogenase, CRP - C-reactive protein, NLR - neutrophil-to-lymphocyte ratio, PLR - platelet-to-lymphocyte ratio, $L M R$ - lymphocyte-to-monocyte ratio.

Table IV. Uni- and multivariate Cox regression analysis for overall survival in patients with Wilms tumour

\begin{tabular}{|lcccc|}
\hline Characteristics & $\begin{array}{c}\text { Univariate } \\
\text { HR }(95 \% \mathrm{Cl})\end{array}$ & $P$-value & $\begin{array}{c}\text { Multivariate } \\
\text { HR }(95 \% \mathrm{Cl})\end{array}$ & $P$-value \\
\hline Age $(<4$ y vs. > 4 y) & $1.18(0.20-7.07)$ & 0.855 & & \\
\hline Stage (4-5 vs. $1-3)$ & $9.38(1.05-83.98)$ & 0.045 & & \\
\hline LDH (H vs. L) & $12.72(1.42-113.81)$ & 0.023 & & \\
\hline CRP (H vs. L) & $19.29(2.15-172.78)$ & 0.008 & $13.40(1.46-123.36)$ & \\
\hline NLR (H vs. L) & $13.11(2.17-79.08)$ & 0.005 & & \\
\hline PLR (H vs. L) & $23.60(3.92-142.07)$ & 0.001 & & 0.018 \\
\hline LMR (L vs. H) & $14.41(1.61-129.29)$ & 0.017 & $20.02(2.12-189.05)$ & \\
\hline
\end{tabular}

$L D H$ - lactate dehydrogenase, CRP - C-reactive protein, NLR - neutrophil-to-lymphocyte ratio, PLR - platelet-to-lymphocyte ratio, $L M R$ - lymphocyte-to-monocyte ratio.

Some paediatric malignancies have a well-established prognostic association with NLR. Nayak et al. found an independent relationship between high NLR and shorter OS in neuroblastoma [17]. Similar findings were observed in paediatric soft tissue sarcomas and salivary malignancies [18, 19]. The limitation of these studies is the variety of histopathological entities included in a single analysis. Other studies demonstrated that DNA methylation-derived NLR may serve as an adverse prognostic factor in medulloblastoma [27]. However, in these studies, the authors did not take into consideration the influence of age on the values of NLR, PLR and LMR. This might have interfered with the outcomes since, during childhood, $\mathrm{CBC}$ norms differ in different age groups. The main change concerns neutrophils and lymphocytes. In- fants up to the $4^{\text {th }}$ day of life have a predominance of neutrophils over lymphocytes. Afterward, the ratio changes, with lymphocytes becoming the major component of the blood leukocytes. Then, around the age of 4 years, neutrophils slowly regain their dominance in the CBC count. Our study demonstrated the prognostic value of age-adjusted ratios of analysed parameters derived from CBC in children with WT. These results support the necessity to perform further studies within children with other malignancies using a similar age-adjusted approach.

The prognostic and predictive value of CBC-derived variables in kidney malignancies was well studied in adult renal cell carcinoma (RCC). Park et al. reported that a lower post-treatment NLR and larger reduction of NLR after sunitinib treat- 
ment were significantly associated with a better response in patients with metastatic RCC [28]. Moreover, Chang et al. analysed the association between preoperative NLR, the postoperative change in NLR and survival in patients who underwent radiofrequency ablation for localized RCC. The study showed that NLR $\geq 2.79$ and a change of NLR $\geq 0.4$ in the postoperative period were associated with poor disease-free survival [29].

Wilms tumour tissues, especially the stromal component, display diminished mitochondrial metabolism with a shift to anaerobic glycolysis and lactate production [30]. Lactate dehydrogenase is a marker of tumour hypoxia and tends to positively correlate with the systemic inflammatory response in solid malignancies in adults [31]. In children with Hodgkin's lymphoma, serum levels of LDH correlated positively with the serum levels of soluble interleukin-2 receptor and reflected the disease stage [32]. High levels of LDH at diagnosis in children with soft tissue sarcomas and Hodgkin's lymphoma were found to decrease in those who responded well to $\mathrm{CHT}[32,33]$. Moreover, elevated LDH is a well-known poor prognostic factor in neuroblastoma [34], Ewing sarcoma [35] and germ cell tumours of childhood [36, 37]. High LDH was reported in WT in both paediatric and adult cases, but its prognostic significance has not yet been established [38].

The usefulness of systemic inflammatory markers in discriminating cancer patients from non-oncological cases has been investigated in several malignancies, including e.g. cervical cancer, and germ cell tumours [39, 40]. Yalon et al. found elevated NLR levels in paediatric patients with CNS tumours compared to the elective control group [20]. Hereby, we report for the first time that patients with WT are characterized by higher levels of hypoxic and systemic inflammatory markers. These measurements performed at diagnosis may serve as ancillary factors in ambiguous cases.

Our study has several limitations. First of all, we present retrospectively collected data, which include a relatively small cohort with a low number of events. In addition, we lack data on the histology of the tumour (i.e. presence of anaplasia), which is a well-established prognostic factor in WT. The control group without malignancy was not composed of patients admitted due to WT suspicion; thus the results of this part of the study should be analysed with caution.

In conclusion, inexpensive and easily accessible preoperative LDH, CRP, NLR, PLR, and LMR have significant prognostic value in patients with WT independently of age and stage. It supports the importance of systemic inflammation in paediatric solid tumours, including WT. In our cohort, combined low CRP and high LMR identified the group of patients with excellent prognosis in terms of OS. On the other hand, patients with high LDH were characterized by the highest risk of relapse. Moreover, patients with WT displayed higher levels of systemic inflammatory markers than children without any malignancy.

\section{Conflict of interest}

The authors declare no conflict of interest.

\section{References}

1. Green DM. The treatment of stages I-IV favorable histology Wilms' tumor. J Clin Oncol 2004; 22: 1366-72.

2. Hol JA, Lopez-Yurda MI, Van Tinteren H, et al. Prognostic significance of age in 5631 patients with Wilms tumour prospectively registered in International Society of Paediatric Oncology (SIOP) 93-01 and 2001. PLoS One 2019; 14: e0221373.

3. Wright KD, Green DM, Daw NC. Late effects of treatment for Wilms tumor. Pediatr Hematol Oncol 2009; 26: 407-13.

4. Kostel Bal AS, Yalcin B, Susam-Şen H, et al. Renal late effects after the treatment of unilateral nonsyndromic Wilms tumor. J Pediatr Hematol Oncol 2016; 38: e147-50.

5. Oostveen RM, Pritchard-Jones K. Pharmacotherapeutic management of Wilms tumor: an update. Pediatr Drugs 2019; 21: 1-13.

6. Coussens LM, Werb Z. Inflammation and cancer. Nature 2002; 420: 860-7.

7. Hanahan D, Weinberg RA. Hallmarks of cancer: the next generation. Cell 2011; 144: 646-74.

8. Toiyama Y, Inoue $\mathrm{Y}$, Kawamura M, et al. Elevated platelet count as predictor of recurrence in rectal cancer patients undergoing preoperative chemoradiotherapy followed by surgery. Int Surg 2015; 100: 199-207.

9. Rao XD, Zhang H, Xu ZS, Cheng H, Shen W, Wang XP. Poor prognostic role of the pretreatment platelet counts in colorectal cancer: a meta-analysis. Medicine 2018; 97: e10831.

10. Wang YH, Kang JK, Zhi YF, et al. The pretreatment thrombocytosis as one of prognostic factors for gastric cancer: a systematic review and meta-analysis. Int J Surg 2018; 53: 304-11.

11. Liu ZQ, Chu L, Fang JM, et al. Prognostic role of C-reactive protein in prostate cancer: a systematic review and meta-analysis. Asian J Androl 2014; 16: 467-71.

12. Yamamoto M, Saito H, Uejima C, et al. Prognostic value of the combination of pre- and postoperative C-reactive protein in colorectal cancer patients. Surg Today 2018; 48: 986-93.

13. Han Y, Mao F, Wu Y, et al. Prognostic role of C-reactive protein in breast cancer: a systematic review and meta-analysis. Int J Biol Markers 2011; 26: 209-15.

14. Guo W, Lu X, Liu Q, et al. Prognostic value of neutrophil-to-lymphocyte ratio and platelet-to-lymphocyte ratio for breast cancer patients: an updated meta-analysis of 17079 individuals. Cancer Med 2019; 8: 4135-48.

15. Diem S, Schmid S, Krapf M, et al. Neutrophil-to-lymphocyte ratio (NLR) and platelet-to-lymphocyte ratio (PLR) as prognostic markers in patients with non-small cell lung cancer (NSCLC) treated with nivolumab. Lung Cancer 2017; 111: 176-81.

16. Zhang J, Zhang HY, Li J, Shao XY, Zhang CX. The elevated NLR, PLR and PLT may predict the prognosis of patients 
with colorectal cancer: a systematic review and meta-analysis. Oncotarget 2017; 8: 68837-46.

17. Nayak A, McDowell DT, Kellie SJ, Karpelowsky J. Elevated preoperative neutrophil-lymphocyte ratio is predictive of a poorer prognosis for pediatric patients with solid tumors. Ann Surg Oncol 2017; 24: 3456-62.

18. Seng D, Fang Q, Li P, Liu F, Liu S. Prognostic value of the pretreatment neutrophil-to-lymphocyte ratio in pediatric parotid cancer. Front Pediatr 2019; 7: 207.

19. Vasquez L, León E, Beltran B, Maza I, Oscanoa M, Geronimo J. Pretreatment neutrophil-to-lymphocyte ratio and lymphocyte recovery: independent prognostic factors for survival in pediatric sarcomas. J Pediatr Hematol Oncol 2017; 39: 538-46.

20. Yalon $M$, Toren A, Jabarin D, Fadida E, Constantini S, Mehrian-Shai R. Elevated NLR may be a feature of pediatric brain cancer patients. Front Oncol 2019; 9: 327.

21. Ray-Coquard I, Cropet C, Van Glabbeke M, et al. Lymphopenia as a prognostic factor for overall survival in advanced carcinomas, sarcomas, and lymphomas. Cancer Res 2009; 69: 5383-91.

22. Begic-Kapetanovic S, Avdagic N, Zaciragic A, Hasic S, Babic N, Hadzimuratovic A. Could the neutrophil-to-lymphocyte ratio serve as a marker in the diagnosis and prediction of acute appendicitis complications in children? Arch Med Sci 2021. DOI: https://doi.org/10.5114/ aoms.2019.87697.

23. Gonzalez H, Hagerling C, Werb Z. Roles of the immune system in cancer: from tumor initiation to metastatic progression. Genes Dev 2018; 32: 1267-84.

24. Templeton AJ, McNamara MG, Šeruga B, et al. Prognostic role of neutrophil-to-lymphocyte ratio in solid tumors: a systematic review and meta-analysis. J Natl Cancer Inst 2014; 106: dju124.

25. Maturu P, Overwijk WW, Hicks J, Ekmekcioglu S, Grimm EA Huff V. Characterization of the inflammatory microenvironment and identification of potential therapeutic targets in Wilms tumors. Transl Oncol 2014; 7: 484-92.

26. Maturu P, Jones D, Ruteshouser EC, et al. Role of cyclooxygenase-2 pathway in creating an immunosuppressive microenvironment and in initiation and progression of Wilms' tumor. Neoplasia 2017; 19: 237-49.

27. Arroyo VM, Lupo PJ, Scheurer ME, et al. Pilot study of DNA methylation-derived neutrophil-to-lymphocyte ratio and survival in pediatric medulloblastoma. Cancer Epidemiol 2019; 59: 71-4.

28. Park YH, Ku JH, Kwak C, Kim HH. Post-treatment neutrophil-to-lymphocyte ratio in predicting prognosis in patients with metastatic clear cell renal cell carcinoma receiving sunitinib as first line therapy. Springerplus 2014; 3: 243.

29. Chang X, Zhang F, Liu T, Wang W, Guo H. Neutrophil-to-lymphocyte ratio as an independent predictor for survival in patients with localized clear cell renal cell carcinoma after radiofrequency ablation: a propensity score matching analysis. Int Urol Nephrol 2017; 49: 967-74.

30. Aminzadeh S, Vidali S, Sperl W, Kofler B, Feichtinger RG. Energy metabolism in neuroblastoma and Wilms tumor. Transl Pediatr 2015; 4: 20-32.

31. Yu SL, Xu LT, Qi Q, et al. Serum lactate dehydrogenase predicts prognosis and correlates with systemic inflammatory response in patients with advanced pancreatic cancer after gemcitabine-based chemotherapy. Sci Rep 2017; 7: 45194

32. Bien E, Balcerska A. Serum soluble interleukin-2 receptor, beta2-microglobulin, lactate dehydrogenase and erythrocyte sedimentation rate in children with Hodgkin's lymphoma. Scand J Immunol 2009; 70: 490-500.

33. Bien E, Rapala M, Krawczyk M, Balcerska A. The serum levels of soluble interleukin-2 receptor $\alpha$ and lactate dehydrogenase but not of B2-Microglobulin correlate with selected clinico-pathological prognostic factors and response to therapy in childhood soft tissue sarcomas. J Cancer Res Clin Oncol 2010; 136: 293-305.

34. Cangemi G, Reggiardo G, Barco S, et al. Prognostic value of ferritin, neuron-specific enolase, lactate dehydrogenase, and urinary and plasmatic catecholamine metabolites in children with neuroblastoma. Onco Targets Ther 2012; 5: 417-23.

35. Li S, Yang Q, Wang H, et al. Prognostic significance of serum lactate dehydrogenase levels in Ewing's sarcoma: a meta-analysis. Mol Clin Oncol 2016; 5: 832-8.

36. Von Eyben FE. A systematic review of lactate dehydrogenase isoenzyme 1 and germ cell tumors. Clin Biochem 2001; 34: 441-54.

37. Kinumaki H, Takeuchi H, Nakamura K, Ohmi K, Bessho F, Kobayashi N. Serum lactate dehydrogenase isoenzyme-1 in children with yolk sac tumor. Cancer 1985; 56: 178-81.

38. Pandian SS, McClinton S, Bissett D, Ewen SWB. Lactate dehydrogenase as a tumour marker in adult Wilm's tumour. Br J Urol 1997; 80: 670-1.

39. Tas M, Yavuz A, Ak M, Ozcelik B. Neutrophil-to-lymphocyte ratio and platelet-to-lymphocyte ratio in discriminating precancerous pathologies from cervical cancer. J Oncol 2019; 2019: 2476082

40. Yuksel OH, Verit A, Sahin A, Urkmez A, Uruc F. White blood cell counts and neutrophil to lymphocyte ratio in the diagnosis of testicular cancer: a simple secondary serum tumor marker. Int Braz J Urol 2016; 42: 53-9. 